\title{
DETERMINANTES E CONDICIONANTES PARAA IMPLEMENTAÇÃO DA CONSULTA DE ENFERMAGEM
}

\author{
Karla Crozeta $^{1}$, Thiago Christel Truppel², Marineli Joaquim Meier ${ }^{3}$, Mitzy Tannia Reichembach Danski ${ }^{4}$
}

\begin{abstract}
RESUMO:Trata-se de uma pesquisa descritiva de abordagem qualitativa desenvolvida no período de 2005 e 2006 , cujo objetivo foi identificar os determinantes e condicionantes para a implementação da consulta de enfermagem a partir das discussões de um grupo focal na perspectiva tecnológica. A utilização do Grupo Focal como técnica de coleta de dados e o referencial da avaliação em saúde subsidiaram a pesquisa. Foram realizadas quatro sessões grupais junto 13 enfermeiros de diversas clínicas de um Hospital Universitário de Curitiba/PR. Os resultados apontaram determinantes e condicionantes relacionados às dimensões estrutura, processo e resultados para a implementação da consulta de enfermagem, tais como: estrutura física inadequada, escassez de recursos humanos, formação inadequada, indisponibilidade de tempo, resolutividade, otimização do serviço, disponibilidade, entre outros. A consulta de enfermagem pode ser uma tecnologia valiosa para o desenvolvimento da autonomia do enfermeiro e proporciona reconhecimento profissional, o que demanda sua constante avaliação.
\end{abstract}

PALAVRAS-CHAVE: Tecnologia; Avaliação em saúde; Enfermagem.

\section{DETERMINANTS TO THE IMPLEMENTATION OF NURSING CONSULTATION}

ABSTRACT: This concerns a descriptive research developed in the period of 2005 and 2006 with a qualitative approach, which has the purpose to identify the determiners and conditionals for the implementation of nursing consultation developed from discussions of a focal group on technological perspectives. The use of the Focal Group as a technique of collecting data and health evaluation background subsidized the research. Four group meetings were held along with nurses from several clinics of a university hospital in Curitiba./PR. The results pointed determiners and conditionals related to dimensions of structure, process, and results for the implementation of a nursing consultation such as: inadequate physical structure, lack of human resources, inadequate undergraduate education, time unavailability, service optimization, among others. Nursing consultation can be a valuable technology for the development of autonomy of a nurse and it fosters professional recognition, demanding constant evaluation by the nurse.

KEYWORDS: Technology; Health evaluation; Nursing.

\section{DETERMINANTES Y CONDICIONANTES PARA LA IMPLEMENTACIÓN DE LA CONSULTA DE ENFERMERÍA}

RESUMEN: Se trata de una investigación descriptiva de abordaje cualitativo desarrollada en el período de 2005 y 2006, que tuvo el objetivo de identificar los determinantes y condicionantes para la implementación de la consulta de enfermería a partir de las discusiones de un grupo focal en la perspectiva de la tecnología. La utilización del Grupo Focal como técnica para recoger datos y el referencial de la evaluación en salud subsidiaron la investigación. Fueron realizados cuatro encuentros grupales con 13 enfermeros de distintas clínicas de un Hospital Universitario de Curitiba/PR. Los resultados apuntaron determinantes y condicionantes relacionados a las dimensiones estructura, proceso y resultados para la implementación de la consulta de enfermería, tales como: estructura física inadecuada, escasez de recursos humanos, formación inadecuada, indisponibilidad de tiempo, capacidad resolutiva, optimización del servicio, disponibilidad, entre otros. La consulta de enfermería puede ser una tecnología valiosa para el desarrollo de la autonomía del enfermero y proporciona reconocimiento profesional, lo que demanda una evaluación constante.

PALABRAS CLAVE: Tecnología; Evaluación en salud; Enfermería.

\footnotetext{
${ }^{1}$ Enfermeira. Mestranda do Programa de Pós-Graduação em Enfermagem da Universidade Federal do Paraná-UFPR. Bolsista CAPES. Membro do Grupo de Estudos Multiprofissional em Saúde do Adulto-GEMSA.

${ }^{2}$ Enfermeiro. Mestrando do Programa de Pós-Graduação em Enfermagem da UFPR. Membro do GEMSA.

${ }^{3}$ Doutora em Filosofia da Enfermagem pela Universidade Federal de Santa Catarina-UFSC. Professora Adjunta do Departamento de Enfermagem e Pós-Graduação em Enfermagem da UFPR. Membro do GEMSA. Orientadora da Pesquisa.

${ }^{4}$ Enfermeira. Doutora em História pela UFPR. Professora Adjunta do Departamento de Enfermagem da UFPR. Membro do GEMSA.
}

Autor correspondente:

Karla Crozeta

Rua Padre Camargo, 280 - 80.060-040 - Curitiba-PR

Recebido: 15/09/08

E-mail:karla_rlf@yahoo.com.br

Aprovado: 29/03/09

Cogitare Enferm 2009 Jan/Mar; 14(1):120-6 


\section{INTRODUÇÃO}

A sociedade atual está envolvida pela tecnologia, cujo desenvolvimento é decorrente do avanço do conhecimento nas diversas áreas. Seu conceito, delineado no contexto histórico, influenciou e influencia as ações, concepções e arranjos sociais, assim como as práticas de saúde e de Enfermagem no Brasil ${ }^{(1)}$. Sua compreensão atual teve início há séculos e relaciona-se às descobertas e progressos científicos da humanidade ${ }^{(2)}$. Neste estudo tecnologia de Enfermagem é entendida como "o conhecimento humano, científico e empírico, sistematizado, que requer a presença humana, visa à qualidade de vida $\mathrm{e}$ se concretiza no ato de cuidar, considerando a questão ética e o processo reflexivo" (1:167).

Nesta perspectiva, a Consulta de Enfermagem (CE) destaca-se como uma tecnologia que contribui para o cuidado efetivo e confere organização das informações de maneira racional, lógica e sistemática ${ }^{(3)}$. Determina progressos na área da saúde por melhorar a estrutura, o processo e os resultados das organizações e serviços, favorecer as medidas de diagnóstico e intervenções e estruturar formas de cuidado eficazes e compatíveis com as necessidades dos indivíduos e comunidade.

A CE é atividade privativa do enfermeiro na qual utiliza diferentes instrumentos de seu processo de trabalho, tais como os diversos padrões de conhecimento (científico, empírico, ético, estético, sócio-político), comunicação, planejamento, criatividade, pensamento crítico e raciocínio clínico.

Infere-se que a CE é estratégia para a aplicação do processo de enfermagem, o que requer competências, conhecimentos, atitudes, habilidades e responsabilidade. Isto permite a identificação, descrição, compreensão e explicação da maneira como os clientes respondem aos problemas de saúde e/ ou processos vitais. Além disso, conduz à identificação dos aspectos que demandam intervenções de enfermagem, a fim de alcançar os resultados pertinentes ao seu processo de trabalho ${ }^{(4)}$. Estas são características importantes para conferir credibilidade e visibilidade ao trabalho do enfermeiro. No entanto, a CE requer avaliação constante para analisar as intervenções, qualidade técnica, cobertura, acesso, equidade, efetividade e eficiência da saúde, assim como a percepção dos usuários a seu respeito ${ }^{(5)}$.

Para realizar esta avaliação em saúde, propôsse uma tríade composta por estrutura, processo e resultado, a fim de direcionar algumas relações entre a importância de avaliar o processo de trabalho e superar os obstáculos.

A dimensão "Estrutura" implica nas características relativamente estáveis dos envolvidos na assistência médica, ou seja, recursos físicos, humanos, materiais e financeiros necessários. A dimensão "Processo" corresponde ao conjunto de atividades desenvolvidas nas relações entre profissionais e pacientes, com base em padrões aceitos sob a perspectiva técnica e administrativa. A dimensão "Resultado" refere-se ao produto final da assistência prestada, às mudanças verificadas no estado de saúde dos pacientes que são atribuídas a um cuidado prévio, incluindo as relacionadas aos conhecimentos e comportamentos, bem como a satisfação do usuário ao cuidado prestado ${ }^{(7)}$.

A importância da avaliação da CE pelo enfermeiro encontra-se na possibilidade de compreender os fatores que a influenciam, a fim de superar os aspectos limitantes, bem como valorizar os aspectos facilitadores e, consequentemente, ampliar a visibilidade do trabalho deste profissional. Complementa-se que a utilização dos princípios de avaliação $^{(6)}$ permite apreender a inserção da CE nas instituições de saúde e direcionar os esforços para eventuais mudanças organizacionais, com o intuito de alcançar a autonomia da Enfermagem.

Percebe-se na instituição em que foi realizado o presente estudo alguns fatores que influenciam de modo positivo e negativo a implementação da CE. Estes são entendidos como determinantes e condicionantes do processo de trabalho do enfermeiro. Os condicionantes referem-se ao que é submisso a certas condições e o "condicionador" denota a regulação e a existência ou o comportamento de algo ou alguém. Em contrapartida, os determinantes indicam, estipulam as causas, fixam com precisão e demarcam limites. Deste modo, são percebidos como indicadores causais da maneira de ser, estar e fazer da profissão(8).

Isto evidencia a importância de reconhecê-los para compreender as influências existentes no trabalho do enfermeiro e, desta forma, elaborar estratégias para superar estes obstáculos. Assim, neste estudo se buscou responder a questão norteadora: quais são os determinantes e condicionantes para a implementação da consulta de enfermagem na prática do enfermeiro? Para respondê-la, delineou-se o seguinte objetivo: identificar os determinantes e condicionantes para a implementação da consulta de enfermagem a partir das 
discussões de um grupo focal na perspectiva tecnológica.

\section{REVISÃO DE LITERATURA}

A tecnologia é um elemento essencial na construção do saber-fazer da saúde e da Enfermagem, uma vez que permeia e influencia suas bases teóricas e práticas, bem como a interação e comunicação humana, a observação, o diagnóstico e as operações regulatórias de sua prática ${ }^{(9)}$. Sua inserção nas instituições de saúde influencia a forma de organização, bem como o agir das pessoas ${ }^{(1)}$. A tecnologia quando incorporada determina normas de conduzir o fazer, integra o cotidiano de trabalho e de produção do saber. Assim, o saberfazer inclui a compreensão do processo de trabalho. A análise deste processo em realidades concretas possibilita a apreensão das contradições e dinâmicas da prática e contribuem com estratégias de mudança ${ }^{(10)}$.

O trabalho é um processo de transformação intencional que ocorre quando o homem tem necessidades a serem satisfeitas ${ }^{(11)}$. A Enfermagem é considerada um trabalho e no próprio desenrolar do processo de trabalho em saúde identifica seus momentos, bem como caracteriza sua finalidade, tecnologia, objeto e agentes ${ }^{(12)}$.

Diante da realidade que se impõe, a compreensão do significado de tecnologia é uma necessidade, bem como o conhecimento de seu uso e implicações para a prática profissional. Com isso, percebe-se a importância de reconhecer a presença e dependência da tecnologia no processo de trabalho.

Conforme exposto, a CE é uma tecnologia privativa do enfermeiro que propicia espaço importante no cenário da saúde, pois caracteriza claramente o que é e o que não é do âmbito da Enfermagem ${ }^{(13)}$. A CE é uma atividade pertinente em âmbito ambulatorial, hospitalar, ocupacional, nas empresas e indústrias, assim como no ambiente domiciliar. Também tem aplicação viável em escolas, asilos, penitenciárias, entre outras instituições, nas quais há a necessidade de atuar na prevenção, proteção e recuperação de agravos ${ }^{(14)}$.

A literatura demonstra que a CE ocupa seu espaço no processo de trabalho do enfermeiro como metodologia da assistência de enfermagem, principalmente em instituições de ensino e no âmbito da pesquisa em enfermagem ${ }^{(7)}$.

\section{METODOLOGIA}

Trata-se de uma pesquisa descritiva de abordagem qualitativa vinculada ao projeto de iniciação científica "Avaliação Tecnológica das Práticas de Cuidar em Enfermagem”. O estudo foi desenvolvido no período de 2005 a 2006 em um Hospital de Ensino da cidade de Curitiba.

A técnica de coleta de dados utilizada foi o Grupo Focal, visto que oferece condições de exploração do tema, bem como promove a compreensão das percepções, sentimentos, atitudes e motivações dos indivíduos ${ }^{(15)}$.

Os participantes desta pesquisa foram 13 enfermeiros de Unidades de Internação e Ambulatórios. A seleção dos integrantes para compor o grupo focal foi realizada por meio dos seguintes critérios: ser enfermeiro lotado nas Unidades de Internação ou Ambulatórios; aceitar participar da pesquisa de modo voluntário e assinar o Termo de Consentimento Livre e Esclarecido (TCLE).

A condução da sessão grupal ocorreu de modo complementar pelo moderador e observador. O primeiro foi responsável pela coordenação do grupo a partir do guia de temas pré-estabelecido. Preparou e instrumentalizou os participantes para favorecer a integração e exposição de idéias, manteve a discussão focalizada e fomentou a expressão de opiniões sobre o tema. O observador colaborou com a condução da sessão grupal, realizou o registro de impressões sobre o grupo, interveio para esclarecer aspectos não abordados pelo moderador e encarregou-se dos aspectos práticos, como o controle do tempo de duração do encontro.

A operacionalização do grupo focal ocorreu por meio do guia de temas e dos seguintes momentoschave: 1) abertura da sessão grupal (apresentações e informações sobre a pesquisa); 2) estabelecimento do setting (aspectos éticos da pesquisa, contrato grupal); 3) debate (centrado no guia de temas elaborado a partir dos objetivos da pesquisa); 4) síntese (validação dos dados) e 5) encerramento (cumprimentos e acertos para o próximo encontro $)^{(1)}$.

A partir do tema selecionado foram propostas quatro sessões grupais com periodicidade quinzenal e duração de 1 hora e 30 minutos cada. A fim de instrumentalizar o grupo para as discussões foi realizado um encontro e uma oficina sobre Metodologia da Assistência/Consulta de Enfermagem, sob a modalidade de evento de extensão.

A análise dos dados foi realizada com base na tríade estrutura/processo/resultados ${ }^{(6)}$, com o intuito de avaliar a CE do Hospital de Ensino e identificar os determinantes e condicionantes para sua implementação. 
Na dimensão "Estrutura" foram agrupados os DC relacionados aos recursos físicos, humanos, materiais e financeiros. Compuseram a dimensão "Processo" os DC que se referem ao conjunto de atividades desenvolvidas na relação entre enfermeiros e pacientes. Por último, considerou-se DC da dimensão "Resultado" as mudanças verificadas no estado de saúde dos pacientes que são atribuídas à $\mathrm{CE}$, bem como a satisfação do usuário ao cuidado prestado.

A pesquisa foi aprovada pelo Comitê de Ética em Pesquisa com Seres Humanos, sob o CAAE
0093.0.208.000-05 e protocolo n 1072/2005.

\section{RESULTADOS}

Os participantes do Grupo Focal discutiram nas cinco sessões os determinantes e condicionantes para a implementação da CE. Os dados coletados foram analisados e categorizados em termos de estrutura, processo e resultado, seguindo o referencial de avaliação em saúde ${ }^{(6)}$. Os resultados são apresentados no Quadro 1 e discutidos a seguir.

Quadro 1 - Determinantes e condicionantes relacionados à estrutura, processo e resultados para a implementação da consulta de enfermagem

\section{Dimensão estrutura Dimensão processo}

Estrutura física inadequada: ausência de Ausência da inserção da CE no espaço ou existência de espaços processo de trabalho do enfermeiro. compartilhados pela equipe multiprofissional.

Aspectos institucionais: políticas, normas e objetivos dos serviços estabelecidos sem a participação do enfermeiro.

Escassez de recursos humanos.

Alta demanda de pacientes.

Poucos enfermeiros que executam a assistência direta.

Perfil profissional não voltado para CE. Indisponibilidade de tempo.

Conhecimento científico: formação inadequado dos enfermeiros sobre a CE.

Falta de apoio da equipe

multiprofissional.

Dificuldade na adaptação do serviço para a realização da $\mathrm{CE}$.

Ausência de discussões para obter reconhecimento dos enfermeiros sobre a importância e relevância da CE.

Poder médico e de administradores no Necessidade de trabalhar em equipe delineamento das políticas institucionais. para entender o que é a $\mathrm{CE}$.

Vinculação do trabalho do enfermeiro ao trabalho do médico.

Desestruturação do trabalho multiprofissional.

Determinantes e condicionantes relacionados à estrutura da consulta de enfermagem

\section{Dimensão resultados}

A CE explicita o escopo de atuação do enfermeiro.

Possibilita o trabalho/relacionamento com a equipe de saúde.

Custo-benefício favorável ao cliente.

Resolubilidade.

Otimização do serviço disponibilizado.

Documentação das informações pertinentes ao paciente.

Garante continuidade da assistência .

Satisfação tanto do cliente (menor retorno, orientações adequadas) como do enfermeiro.

Reconhecimento pessoal e profissional do enfermeiro. 
da CE, tais como estrutura física, aspectos institucionais, escassez de recursos humanos, alta demanda de pacientes, conhecimento científico insuficiente, falta de apoio da equipe multiprofissional, desestruturação do trabalho multiprofissional, vinculação do trabalho do enfermeiro ao trabalho do médico, entre outros.

No que se refere à estrutura física, os enfermeiros evidenciaram a ausência de um ambiente específico para a realização da CE ou a existência de espaços compartilhados pela equipe multiprofissional. Este é um fator limitante para a implementação da CE nesta instituição, possivelmente gerado por tratarse de uma instituição pública de grande porte e alta complexidade com instalações prediais antigas.

Os aspectos institucionais aparecem como determinantes/condicionantes que dificultam a operacionalização da CE, uma vez que as políticas, normas e objetivos da organização muitas vezes não privilegiam o trabalho desenvolvido pelo enfermeiro por ser visto como mero executor de tarefas rotineiras. Isto se deve ao fato de que estas "políticas, normas e objetivos dos serviços são estabelecidos por médicos e administradores sem a participação dos enfermeiros"(16:52). Portanto, evidencia-se que participar politicamente é uma dimensão do processo de trabalho da enfermagem a ser explorada pelos integrantes da profissão, a fim de conquistarem espaços nos cenários de atenção à saúde.

A escassez de recursos humanos, alta demanda de pacientes, o dimensionamento inadequado de enfermeiros para o cuidado direto e o perfil profissional incompatível para a realização da CE foram apontados como determinantes/condicionantes que dificultam a implementação desta tecnologia, visto que se trata de uma atividade que demanda tempo e disponibilidade para sua realização, assim como conhecimentos específicos.

Quanto a estes conhecimentos científicos o grupo enfatizou sua importância para a implementação da CE. Discutiu a formação do enfermeiro e destacou que o tema não é enfatizado durante o curso de graduação. Isto corrobora os dados da literatura, visto que em cursos de especialização são comuns os relatos de alunos sobre a ausência da utilização da CE durante a formação profissional ${ }^{(13)}$.

Quanto à falta de apoio da equipe multiprofissional, a ideologia médica e as estruturas de poder caracterizam as instituições, o que reforça o poder do médico e, recentemente, dos administradores. A enfermagem se caracteriza por uma ambigüidade, uma vez que de um lado ocupa a posição de cuidado e de outro, o compromisso com a instituição ${ }^{(16)}$.

O grupo reforça esta vinculação do trabalho do enfermeiro ao trabalho do médico e afirma que é necessária a reformulação do sistema hospitalar para que esta realidade seja modificada. Além disso, apontou que os diferentes profissionais da equipe de saúde têm dificuldade em perceber a necessidade de encaminhamento para outro profissional, o que resulta em desestruturação do trabalho multiprofissional.

Assim, as dificuldades para a realização da CE ressaltadas pelos enfermeiros do grupo focal reiteram os obstáculos apontados na literatura e incluem: a escolha, a interpretação e a aplicação do modelo conceitual, além de sua operacionalização; espaço limitado de ação e criatividade imposto à Enfermagem por políticas de outros profissionais; recursos humanos insuficientes; conhecimento científico deficiente; teoria e prática de Enfermagem dissociadas; e modelo gerencial das instituições que aumentam o poder médico e dos administradores enquanto depreciam o da Enfermagem ${ }^{(16)}$.

\section{Determinantes e condicionantes relacionados ao processo da consulta de enfermagem}

No âmbito do processo foram apontados como determinantes/condicionantes: ausência da inserção da CE no processo de trabalho, execução de funções não privativas e inerentes ao cargo, aceitação e acomodação dos enfermeiros em relação às determinações das instituições quanto às suas funções, impossibilidade de delegar a $\mathrm{CE}$ a outros profissionais, indisponibilidade de tempo para sua execução, estabelecimento da CE como atividade prioritária, competência profissional, ausência de discussões sobre $\mathrm{CE}$ e necessidade de trabalho em equipe.

O processo de trabalho do enfermeiro representa a articulação de elementos que caracterizam o saber/ fazer da profissão. Estes estão intrinsecamente relacionados ao trabalho da saúde em seu contexto geral. Além de seus elementos, o trabalho do enfermeiro é permeado por dimensões práticas, as quais são descritas como cuidar, educar, gerenciar e pesquisar ${ }^{(8)}$.

O grupo apontou que a inserção da CE como instrumento do processo de trabalho do enfermeiro não ocorre, pois este assume inúmeros papéis administrativos e institucionais, o que compromete $o$ desenvolvimento de suas atividades privativas. Nas discussões, os participantes salientaram que ocorre o 
"fenômeno da acomodação" (termo utilizado pelo grupo), no qual o profissional aceita esta influência institucional como fator pertencente ao seu processo de trabalho e assume os diversos papéis determinados pelos serviços de saúde, muitas vezes não inerentes ao cargo assumido.

Outro aspecto enfatizado pelo grupo que determina que a CE deva ser executada pelo enfermeiro é a impossibilidade de delegar sua realização ao auxiliar ou técnico de enfermagem, visto que se trata de atividade privativa estabelecida pela lei do exercício profissional. Isto denota que os enfermeiros conhecem suas responsabilidades legais e que é necessário priorizar estas atividades para produzir e valorizar o trabalho deste profissional, assim como aumentar sua visibilidade e autonomia.

A indisponibilidade de tempo foi outro ponto abordado pelo grupo, porém as discussões apontaram para a necessidade do planejamento das atividades. O planejamento é fundamental para as ações do enfermeiro, uma vez que representa a base de todas as atividades. Sua aplicabilidade está relacionada tanto à assistência direta ao cliente como a atividades administrativas, organização do serviço, recursos físicos, materiais e humanos ${ }^{(17)}$.

Quanto à dificuldade na adaptação do serviço à sua realização, o grupo relata que há diferença entre os setores e indica que estas diferenças dependem dos fatores apontados como limitantes e facilitadores da CE: espaço físico, trabalho em equipe e recursos humanos. Esta reflexão conduziu a discussão das possibilidades de implementação da tecnologia, da necessidade de estabelecê-la como prioridade e realizála para conquistar espaço na equipe multiprofissional, bem como reconhecimento institucional.

O grupo conduziu uma das reflexões para o estabelecimento de estratégias e afirmou que faltam discussões sobre o tema e trabalho em equipe, a fim de obter o reconhecimento dos profissionais sobre a importância da CE. Como estratégia de alcance deste reconhecimento está a promoção de encontros, seminários e divulgação da tecnologia em meios científicos.

\section{Determinantes e condicionantes relacionados aos resultados da consulta de enfermagem}

Como determinantes/condicionantes foram citados: a CE esclarece o escopo de atuação do enfermeiro, possibilita o trabalho com a equipe de saúde, possibilita o custo-benefício favorável ao cliente, apresenta resolubilidade nos aspectos relacionados à saúde, otimiza o atendimento oferecido, permite a documentação do cuidado oferecido ao paciente, garante a continuidade da assistência e amplia a satisfação tanto do cliente (menor retorno, orientações adequadas) como do enfermeiro, pelo reconhecimento pessoal e profissional.

A CE foi apontada pelos participantes como um dos métodos de esclarecimento da atuação do enfermeiro e compartilhamento com a equipe de saúde. Sobre a autonomia, o grupo apontou a necessidade deste profissional aprofundar seus conhecimentos científicos para obter sua valorização perante a equipe. As bases teóricas guiam e aprimoram a prática profissional, e, por conseguinte, o desenvolvimento teórico influencia o nível de conhecimento e a formação dos profissionais, tanto na pesquisa quanto na prática da enfermagem ${ }^{(17)}$.

Quanto às vantagens foram elencadas: gratificação pessoal e diminuição do retorno do paciente após a alta hospitalar devido à otimização do serviço disponibilizado e maior resolutividade das necessidades de saúde. Além disso, foi destacada a importância de registrar corretamente os dados no prontuário do paciente, pois esta troca de informações é fundamental e a sua realização inadequada compromete a determinação dos diagnósticos de enfermagem, bem como outras etapas do processo.

O registro das informações fornece elementos para as próximas consultas, contribui para a continuidade da assistência sistemática, bem como beneficia o cliente e o enfermeiro na sua realização ${ }^{(13)}$.

\section{CONSIDERAÇÕES FINAIS}

O presente estudo respondeu a questão problema e alcançou o objetivo proposto, ou seja, identificaram-se os determinantes e condicionantes para a implementação da CE. Sua compreensão ocorreu por meio do grupo focal que possibilitou melhor entendimento sobre o processo de trabalho do enfermeiro, suas implicações e influências.

A tecnologia foi apontada como aplicação do conhecimento científico no campo prático, uma vez que se enfatizou a importância do conhecimento especializado na área de atuação e da competência para desenvolver a consulta, do trabalho interdisciplinar, da disponibilidade, do interesse, da visão integral e da sensibilidade, o que confirma a descrição da CE como uma tecnologia. 
A análise dos dados permitiu apontar os determinantes e condicionantes inerentes à CE. Estes deverão ser trabalhados com vistas à superação e incorporação desta tecnologia no processo de trabalho do enfermeiro.

A implantação da CE requer mudanças na prática do enfermeiro que precisa perceber a complexidade da mesma, uma vez que esta deve estar fundamentada em metodologia própria ${ }^{(3)}$. Portanto, o enfermeiro necessita desempenhar atividades sistematizadas, com objetivos de detectar desvios de saúde e, principalmente, de manter o padrão saudável de cada indivíduo.

\section{REFERÊNCIAS}

1. Meier MJ. Tecnologia em enfermagem: desenvolvimento de um conceito [tese]. Florianópolis (SC): Universidade Federal de Santa Catarina; 2004.

2. Grinspun MPSZ. Educação tecnológica: desafios e perspectivas. São Paulo: Cortez; 1999.

3. Zagonel IPS. Consulta de Enfermagem: um modelo de metodologia para o cuidado. In: Westphalen MEA; Carraro TE, organizadores. Metodologias para assistência de enfermagem: teorizações e subsídios para a prática. Goiânia: AB; 2001. p. 41-56.

4. Garcia TR, Nóbrega MML, Carvalho EC. Nursing process: application to the professional practice. Online Brazilian Journal of Nursing [Periódico na Internet]. 2004 3(2) [acesso 2008 Ago 24]. Disponível:www.uff.br/ nepae/objn302garciaetal.htm

5. Silva LMV. Conceitos, abordagens e estratégias para a avaliação em saúde. In: Hartz ZMA; Silva LMV, organizadores. Avaliação em saúde: dos modelos teóricos à prática na avaliação de programas e sistemas de saúde. Salvador/Rio de Janeiro: Fiocruz/UFBA; 2005.

6. Donabedian A. Defining and measuring the quality of health care. In: Wenzel RP. Assessing quality of health /care-perspective for clinicians. Baltimore: Williams \& Wilks; 1982. p.41-64.

7. Silva LMV, Formigli VLA. Avaliação em saúde: limites e perspectivas. Cad Saúde Publ. 1994; 10(1):80-91.

8. Taube SAM. O processo de trabalho da enfermeira na central de material e esterilização: uma perspectiva tecnológica aos instrumentos [dissertação]. Curitiba (PR): Universidade Federal do Paraná; 2006.

9. Barnard A. A critical review of the belief that technology is a neutral object and nurses are its master. J Advanced Nurs. 1997;26:126-31.

10. Orem DE. Nursing concepts of practice. EUA: MosbyYear Book; 1995.

11. Peduzzi M, Anselmi ML. O processo de trabalho de enfermagem: cisão entre planejamento e execução do cuidado. Rev Bras Enferm. 2002; 55(4):393-98.

12. Almeida MCP; Rocha SMM, organizadores. O trabalho de enfermagem. São Paulo: Cortez; 1997.

13. Vanzin AS, Nery ME. Consulta de Enfermagem: uma necessidade social? Porto Alegre: MeL Gráfica e Editora; 2000.193p.

14. Rosal LM, Mercês NNA, Marcelino SR, Radünz V. A consulta de enfermagem no cuidado à pessoa com câncer: contextualizando uma realidade. Cogitare Enferm. 2007 Out/Dez; 12(4):487-93.

15. Edmunds $\mathrm{H}$. The focus group research handbook. EUA: Mc Graw-Hill; 1999.

16. Rossi LA, Casagrande LD. Processo de enfermagem: a ideologia da rotina e a utopia do cuidado individualizado. In: Cianciarullo TI, organizador. Sistemas de assistência de enfermagem: evolução e tendências. São Paulo: Ícone; 2001. p.29-39.

17. Ciampone MHT. Metodologia do planejamento na enfermagem. In: Kurcgant P. Administração em enfermagem. São Paulo: EPU; 1991. 